\title{
Tunable interfaces for realizing universal quantum computation with topological qubits
}

\author{
Zheng-Yuan Xue, ${ }^{1,2, *}$ L. B. Shao, ${ }^{3}$ Yong Hu, ${ }^{1,4}$ Shi-Liang Zhu, ${ }^{3,2}$ and Z. D. Wang ${ }^{1, \dagger}$ \\ ${ }^{1}$ Department of Physics and Center of Theoretical and Computational Physics, The University of Hong Kong, \\ Pokfulam Road, Hong Kong, China \\ ${ }^{2}$ Laboratory of Quantum Engineering and Quantum Materials, and School of Physics and Telecommunication Engineering, \\ South China Normal University, Guangzhou 510006, China \\ ${ }^{3}$ National Laboratory of Solid State Microstructure and Department of Physics, Nanjing University, Nanjing 210093, China \\ ${ }^{4}$ School of Physics, Huazhong University of Science and Technology, Wuhan 430074, China
}

(Received 8 May 2013; published 20 August 2013)

\begin{abstract}
We propose to implement tunable interfaces for realizing universal quantum computation with topological qubits. One interface is between the topological and superconducting qubits, which can realize an arbitrary single-qubit gate on the topological qubit. When two qubits are involved, the interface between the topological qubits and a microwave cavity can induce a nontrivial two-qubit gate, which cannot be constructed based on braiding operations. The two interfaces, being tunable via an external magnetic flux, may serve as the building blocks towards universal quantum computation with topological qubits.
\end{abstract}

DOI: 10.1103/PhysRevA.88.024303

PACS number(s): 03.67.Lx, 42.50.Dv, 74.78.Na

Topological qubits are largely insensitive to local noises [1] and thus hold a promising future in quantum information processing. For universal quantum computation, one needs to encode a topological qubit with non-Abelian anyons [2]. Therefore, Majorana fermions (MFs) with non-Abelian statistics have recently attracted strong renewed interest [3]. MFs are a kind of self-conjugate quasiparticle proposed in some systems theoretically, e.g., certain vortex excitations in chiral $p$-wave superconductors [2]. However, an unambiguous experimental verification of MFs is still awaited. Recently, it is indicated theoretically that MFs can be created on the interface between a strong topological insulator (TI) [4] and an $s$-wave superconductor by the proximity effect [5]. Similar schemes with spin-obit coupling and $s$-wave pairing have also been proposed [6-12], which have greatly advanced the field. Meanwhile, the interfaces between topological qubits and quantum dots [13-16], as well as superconducting qubits [17-23], have also been proposed. These hybrid systems may allow us to consolidate the advantages of both types of qubits.

In this Brief Report, we propose tunable interfaces for realizing universal quantum computation with topological qubits. Here, the hybrid system is constructed with a topological qubit, a superconducting charge qubit, and a microwave cavity. In addition to an external magnetic flux, we also introduce a cavity-induced magnetic flux in the superconducting qubit loop. In this way, an interface between the topological qubit and the cavity, mediated by the superconducting qubit, may be implemented. By modulating the external magnetic flux, the interfaces between the topological qubit and the superconducting qubit or the cavity can be switched on alternatively. For universal quantum computation, the interface between the topological and the superconducting qubits is sufficient for single-qubit control over and read out of the topological qubit, noting that a topological qubit is usually hard to be read out. Another difficulty of quantum computation with topological

\footnotetext{
*zyxue@scnu.edu.cn

†zwang@hku.hk
}

qubits lies in the fact that braiding cannot implement a nontrivial two-qubit gate. However, in our proposal, when two qubits are involved, the interface between the topological qubits and a microwave cavity can induce a nontrivial twoqubit gate. Therefore, the two interfaces may serve as the building blocks towards universal quantum computation with topological qubits.

We now proceed to introduce our considered setup. First, the topological qubit is encoded by four MFs $\left\{\gamma_{i}\right\}_{i=1,2,3,4}$, which satisfy the fermionic anticommutation relation. A Dirac fermion can be constructed from a pair of MFs $\tilde{c}_{i j}^{\dagger}=\left(\gamma_{i}-i \gamma_{j}\right) / \sqrt{2}$, defining a twofold degenerated Hilbert space labeled by the fermion parity $n_{i j}=\tilde{c}_{i j}^{\dagger} \tilde{c}_{i j}=0,1$. In the even-parity subspace, a topological qubit is encoded with the basis states $|0\rangle_{t}=|0\rangle_{12}|0\rangle_{34}$ and $|1\rangle_{t}=|1\rangle_{12}|1\rangle_{34}$, where the subscript $t$ denotes that the state is of the topological qubit. The MFs can be created on the surface of a TI patterned with $s$-wave superconductors [5], and thus the Cooper pairs can tunnel into the TI due to the proximity effect. Then, assuming that the chemical potential is in the vicinity of the Dirac point, the Hamiltonian of the surface will obtain an additional $s$-wave pairing term. As shown in Fig. 1, each MF, indicated by a red circle, is localized at a point where three separated superconducting islands meet, i.e., a superconducting trijunction. The MFs can be created via a superconductor-TI-superconductor (STIS) wire that separates the islands with superconducting phases $\phi_{d}=\varepsilon$ and $\phi_{u}=-\pi$. A narrow STIS wire (width $W \ll v_{F} / \Delta_{0}$ ) is described by [5]

$$
H_{\mathrm{STIS}}=-i v_{F} \tilde{\tau}_{x} \partial_{x}+\delta_{\varepsilon} \tilde{\tau}_{z}
$$

where $v_{F}$ is the effective Fermi velocity, $\Delta_{0}$ is the $s$-wave superconducting gap, $\delta_{\varepsilon}=\Delta_{0} \cos \left[\left(\phi_{d}-\phi_{u}\right) / 2\right]=$ $-\Delta_{0} \sin \varepsilon / 2$, and $\tilde{\tau}_{x, z}$ are the Pauli matrices acting on the wire's zero modes. Figure 1 shows two pairs of MFs with distance $L$, which encode our topological qubit. The two pairs of MFs share the same type of coupling; e.g., for $\gamma_{1}$ and $\gamma_{2}$ the coupled Hamiltonian is $\tilde{H}_{12}^{\mathrm{MF}}=i E(\varepsilon) \gamma_{1} \gamma_{2} / 2$ with an energy splitting $E(\varepsilon)$ depending on the superconducting phase $\phi_{d}=\varepsilon$. An 


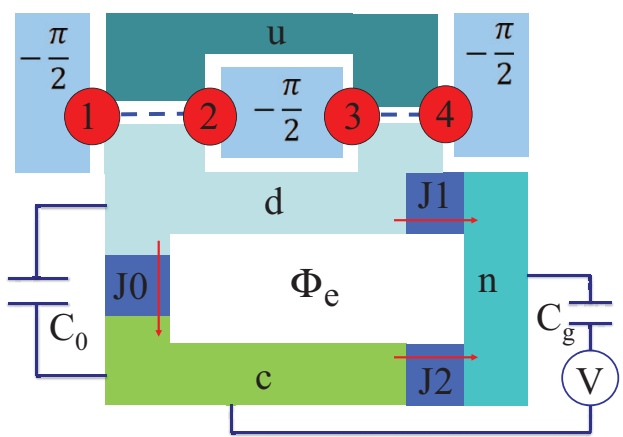

FIG. 1. (Color online) On the surface of TI, patterned superconducting islands can form hybrid system of topological and superconducting qubits. Two pairs of MFs (red circles) are localized at superconducting trijunctions, connected by STIS quantum wires (dashed blue line). The coupling between a pair of MFs is controlled by the superconducting phases $\phi_{d}=\varepsilon$ and $\phi_{u}=-\pi$. The superconducting charge qubit consists of three JJs, enclosing external magnetic fluxes from a dc magnetic field and a microwave cavity field (not shown). The superconducting phase $\phi_{c}$ is fixed relative to $\phi_{u}$ by a phase controller (not shown). Then, $\phi_{d}$ will depend on the state of the superconducting qubit and is related to the cavity field.

effective Hamiltonian for the topological qubit is

$$
H_{T}=-\frac{E(\varepsilon)}{2} \tau_{z}
$$

where $\tau_{z}=(|0\rangle\langle 0|-| 1\rangle\langle 1|)_{\mathrm{t}}$ is the Pauli matrix acting on the topological qubit and

$$
E(\varepsilon)=\frac{v_{F}}{L} \sqrt{\Lambda_{\varepsilon}^{2}+f_{0}^{2}\left(\Lambda_{\varepsilon}\right)},
$$

with the dimensionless parameter $\Lambda_{\varepsilon}=\frac{\Delta_{0} L}{v_{F}} \sin \frac{\varepsilon}{2}$ and $f_{n}(y)$ (with $n=0,1,2, \ldots$ ) being the inverse function of $y=$ $x / \tan (x)$ associated with the $n$th invertible domain. For $\Lambda_{\varepsilon} \gg 1$ and $0<\varepsilon<\pi / 2$, the topological qubit splitting $E(\varepsilon)$ is negligibly small [5]. While for $\Lambda_{\varepsilon} \leqslant 1, E(\varepsilon)$ becomes sensitive to $\varepsilon$. To couple the topological and superconducting qubits, it is natural to make $\varepsilon$ dependent on the superconducting qubit state. As shown in Fig. 1, this can be achieved by making the superconductor labeled " $\mathrm{d}$ " be a part of the superconducting qubit, and thus $\varepsilon$ is related to the magnetic flux pierced in the qubit loop. Meanwhile, the superconducting qubit is placed in a cavity, and thus the magnetic flux contains the external magnetic flux and the magnetic flux coming from the cavity. In this way, coupling among the three elements can be implemented.

We now detail the coupling of the elements. As also shown in Fig. 1, the superconducting charge qubit [24-26] consists of a small superconducting box with $n$ excess Cooper-pair charges, formed by a symmetric superconducting quantum interference device including two small identical Josephson junctions (JJs) with capacitance $C_{J}$ and Josephson coupling energy $E_{J}$ and pierced by an external magnetic flux $\Phi_{e}$. Meanwhile, a control gate voltage $V$ is applied via a gate capacitor $C_{g}$. To slightly modulate the superconducting phase $\phi_{d}$, a large $\mathrm{JJ}$ is also introduced, which has a Josephson coupling energy of $E_{J 0} \gg E_{J}$ and a capacitance of $C_{J 0}$. In order to eliminate the influence of the charging energy of the large JJ to the superconducting charge qubit Hamiltonian, a large capacitance $C_{0}$ is placed in parallel with the large $\mathrm{JJ}$ [24]. Assuming that the inductance of the qubit circuit is much smaller than that of the large JJ, the Hamiltonian of the superconducting qubit can be written as [26]

$$
H_{S}=E_{c}\left(n-n_{g}\right)^{2}-E_{J}\left(\cos \phi_{1}+\cos \phi_{2}\right)-E_{J 0} \cos \phi_{J},
$$

where $E_{c}=2 e^{2} /\left(C_{g}+2 C_{J}\right)$ is the charging energy of the superconducting island, $n_{g}=C_{g} V /(2 e)$ is the induced charge of the gate voltage, and $\phi_{J}, \phi_{1}$, and $\phi_{2}$ are the phase drops across JJs 0,1 , and 2 , respectively.

Meanwhile, the superconducting charge qubit is placed at a magnetic antinode of the cavity in a circuit QED scenario [27]. For simplicity, we assume that the cavity has only a single mode to play a role, the free Hamiltonian of which is $H_{c}=\omega_{r} a^{\dagger} a$ (assuming $\hbar=1$ hereafter) with $\omega_{r}, a$, and $a^{\dagger}$ being the frequency, annihilation, and creation operators of the cavity mode, respectively. Flux quantization around the qubit loop leads to $\phi_{1}=\phi-\beta$ and $\phi_{2}=\phi+\beta$, where $2 \beta=\phi_{e}-\phi_{J}+2 g\left(a+a^{\dagger}\right)$ with $\phi_{e}=2 \pi \Phi_{e} / \Phi_{0}$ and $g$ is the magnetic coupling strength between the cavity and the superconducting qubit; the average phase drop $\phi=\left(\phi_{1}+\right.$ $\left.\phi_{2}\right) / 2$ is canonically conjugate to $n$ as $[\phi, n]=i$. Consequently, the qubit Hamiltonian in Eq. (4) can be rewritten as [26]

$$
H_{C S}=E_{c}\left(n-n_{g}\right)^{2}-2 E_{J} \cos \beta \cos \phi-E_{J 0} \cos \phi_{J} .
$$

The induced circulating supercurrent in the qubit circuit is $I=2 I_{c} \sin \beta \cos \phi$, with $I_{c}=\pi E_{J} / \Phi_{0}$ being the critical current of the two small JJs. Meanwhile, this supercurrent also flows through the large JJ, and thus $I=I_{0} \sin \phi_{J}$, with $I_{0}=2 \pi E_{J 0} / \Phi_{0}$ being the critical current of the large JJ. Therefore,

$$
I_{0} \sin \phi_{J}=2 I_{c} \sin \beta \cos \phi .
$$

As $E_{J} \ll E_{J 0}, \phi_{J}$ will be small. Up to the second order of the small parameter of $\eta=I_{c} / I_{0}$, we have

$$
\begin{aligned}
\phi_{J}= & 2 \eta \sin \frac{\phi_{e}}{2} \cos \phi-\eta^{2} \sin \phi_{e} \cos ^{2} \phi \\
& +2 g \eta \cos \frac{\phi_{e}}{2} \cos \phi \times\left(a+a^{\dagger}\right) .
\end{aligned}
$$

At low temperatures $\left(k_{B} T \ll E_{c}\right)$ and within the charging regime $\left(E_{J} \ll E_{c} \ll \Delta_{0}\right)$, only the lowest two charge states $\left\{|0\rangle_{s},|1\rangle_{s}\right\}$ are relevant for the superconducting qubit operating at its degeneracy point $\left(n_{g}=1 / 2\right)$, where the subscript $s$ denotes that the state is of the superconducting qubit hereafter. As a result, the Hamiltonian in Eq. (5) reduces to

$$
H_{C S C}=-\frac{\bar{E}_{J}}{2} \sigma_{x}+\xi\left(a+a^{\dagger}\right) \sigma_{x},
$$

where $\quad \bar{E}_{J}=2 E_{J} \cos \frac{\phi_{e}}{2}\left(1-\frac{3}{8} \eta^{2} \sin ^{2} \frac{\phi_{e}}{2}\right), \quad \xi=g E_{J} \sin \frac{\phi_{e}}{2}$, and $\sigma_{x, z}$ are Pauli matrices acting on the superconducting qubit state. Meanwhile, in the superconducting qubit representation, $\phi_{J}=f_{1}+\left(f_{2}+f_{3}\right) \sigma_{x}$, where $f_{1}=-\frac{1}{4} \eta^{2} \sin \phi_{e}$, $f_{2}=\eta \sin \frac{\phi_{e}}{2}$, and $f_{3}=\eta g\left(a+a^{\dagger}\right) \cos \frac{\phi_{e}}{2}$, which depends on the states of the superconducting charge qubit and the cavity. If we fix $\phi_{c}$ with respect to $\phi_{u}$ with a phase controller, up to the second order of $\eta, \phi_{d}$ will be $\varepsilon^{+}=\phi_{c}+f_{1}+f_{2}+f_{3}$ 
and $\varepsilon^{-}=\phi_{c}+f_{1}-f_{2}-f_{3}$, depending on the state of the superconducting charge qubit in the states $|+\rangle_{s}$ and $|-\rangle_{s}$, respectively. As $\eta$ is small, the separation of $\phi_{d}, \Delta \varepsilon=$ $2\left(f_{2}+f_{3}\right) \propto \eta$, will be small, as we expected.

Finally, the combined hybrid system can be described by $H_{\text {total }}=\omega_{r} a^{\dagger} a-\frac{1}{2} \omega_{t} \tau_{z}+H_{C S C}+H_{\text {int }}$, with the interaction between the topological qubit and others being

$$
H_{\mathrm{int}}=-\frac{\lambda_{1}}{2} \sigma_{x} \tau_{z}-\lambda_{2} \sigma_{x} \tau_{z}\left(a+a^{\dagger}\right),
$$

where $\omega_{t}=E\left(\phi_{c}+f_{1}\right), \lambda_{1}=\left.\eta \sin \frac{\phi_{e}}{2} \frac{d E(\phi)}{d \phi}\right|_{\phi=\phi_{c}+f_{1}}$, and $\lambda_{2}=$ $\left.\eta g \cos \frac{\phi_{e}}{2} \frac{d E(\phi)}{d \phi}\right|_{\phi=\phi_{c}+f_{1}}$. It is obvious that $\lambda_{1,2}$ can be tuned via the external magnetic flux $\Phi_{e}$. In particular, when $\left|\lambda_{1}\right|$ $\left(\left|\lambda_{2}\right|\right)$ reaches its maximum value, $\lambda_{2}\left(\lambda_{1}\right)$ will be 0 . That is to say, we can selectively implement the topological and superconducting qubits interface or the topological qubit and cavity interface. This is distinctly different from the proposed interface in Ref. [21], where the only implemented interface is between the topological and superconducting flux qubits.

We now consider the interface between the topological and superconducting charge qubits which can be switched on by modulating the external magnetic flux to fulfill $\sin \frac{\phi_{e}}{2}=1$ $\left(\cos \frac{\phi_{e}}{2}=0\right)$. With $\lambda_{1} t_{1}=-\pi / 2$, up to local rotations on the superconducting qubit and Hadamard gates on the topological qubit, we can implement arbitrary unitary transformations for the two-qubit hybrid system [28-30]. For universal quantum computation, this interface is sufficient for single-qubit control over and read out of the topological qubit.

We now estimate the coupling strength of the interface with typical experimental parameters. For the superconducting qubit, we may choose the following parameters [25]: $E_{J}=$ $16 \mathrm{GHz}$ and $E_{J 0}=10 E_{J}$, which means $\eta=0.1$. For the topological qubit, reasonable parameters are the following [21,24]: $\Delta_{0}=2 \pi \times 32 \mathrm{GHz}, v_{F}=10^{5} \mathrm{~m} / \mathrm{s}, L=5 \mu \mathrm{m}$, and $T=20 \mathrm{mK}$. Therefore, the maximum coupling strength of this interface is $\lambda_{1}^{\max } \approx \eta \Delta_{0}=2 \pi \times 3.2 \mathrm{GHz}$.

The relevant imperfections of this interface are estimated as the following. First, as $\lambda_{1} /\left(2 E_{J}\right)=0.1$, the undesired tunneling probability between the qubit states is suppressed to $P_{t} \sim 0.01$. Second, to suppress the quantum fluctuations of the large $\mathrm{JJ}, C_{0}=100\left(C_{g}+2 C_{J}\right)$ is chosen to make its effective charging energy negligible small [25], and thus it works in the classical regime [31]. Finally, excitation of the quantum wire modes with energy $E \approx v_{F} / L$ can be exponentially suppressed to $P_{e} \sim \exp \left[-E /\left(K_{B} T\right)\right]<10^{-3}[21]$.

We move to the topological qubit and microwave cavity interface by modulating $\cos \frac{\phi_{e}}{2}=1$. The coupling between these two subsystems is mediated by the superconducting charge qubit. Without loss of generality, we assume that the superconducting qubit is initially prepared in its ground state. We further tune the energy splitting of the superconducting charge qubit far away from the cavity frequency so that the superconducting qubit will always stay in its ground state. When two identical qubits are involved, the hybrid system is described by the interaction

$$
\begin{aligned}
H_{C T}= & \omega_{r} a^{\dagger} a-\frac{1}{2}\left(\hbar \omega_{t}+\lambda_{1}\right)\left(\tau_{1}^{z}+\tau_{2}^{z}\right) \\
& -\lambda_{2}\left(\tau_{1}^{z}+\tau_{2}^{z}\right)\left(a+a^{\dagger}\right)
\end{aligned}
$$

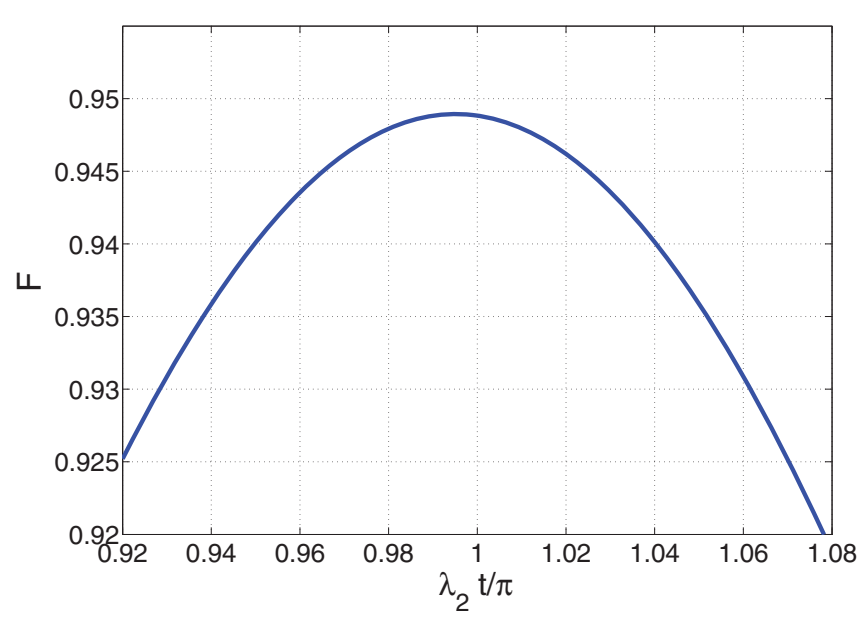

FIG. 2. (Color online) The fidelity of entangling the two topological qubits as a function of $\lambda_{2} t / \pi$. The parameters are $k=1$, $\kappa=\gamma=1 \mathrm{MHz}$, and $\lambda_{2}=2 \pi \times 32 \mathrm{MHz}$.

Setting $\phi_{e}^{\prime}=2 \omega t+\phi_{e}$, the interaction Hamiltonian in the interaction picture reads [32]

$$
H_{I}=-\lambda_{2}\left(a e^{-i v t}+a^{\dagger} e^{i v t}\right) J_{z}
$$

where $v=\omega_{r}-\omega>0$ and $J_{z}=\sum_{j} \tau_{j}^{z} / 2$. The timeevolution operator for the Hamiltonian in Eq. (11) can be expressed as [33,34]

$$
\begin{aligned}
U(t)= & \exp \left[-i A(t) J_{z}^{2}\right] \\
& \times \exp \left[-i B(t) a J_{z}\right] \exp \left[-i B^{*}(t) a^{\dagger} J_{z}\right],
\end{aligned}
$$

where

$$
\begin{gathered}
A(t)=-\frac{\lambda_{2}^{2}}{v}\left[t-\frac{1}{i v}\left(e^{i v t}-1\right)\right], \\
B(t)=-i \frac{\lambda_{2}}{v}\left(e^{-i v t}-1\right) .
\end{gathered}
$$

It is obvious that $B(t)$ is a periodic function of time and vanishes at $\nu \tau=2 k \pi$, where $k=1,2,3, \ldots$. At this time, the operator in Eq. (12) reduces to

$$
U(\tau)=\exp \left[-i A(\tau) J_{z}^{2}\right]
$$

with $A(\tau)=-\lambda_{2}^{2} \tau / \nu$. The maximum coupling strength of this interface is $\lambda_{2}^{\max } \approx \eta g \Delta_{0}=2 \pi \times 32 \mathrm{MHz}$ for $g=0.01$. In this way, we achieve the coupling between the topological qubits mediated by the microwave cavity and the operator in Eq. (15) serves as a nontrivial two-qubit gate.

For example, choosing $A(\tau)=-\pi / 2$ and the initial state of the two topological qubits being $|\psi\rangle_{i}=|++\rangle_{t}$, the final state is

$$
|\psi\rangle_{f}=\frac{1}{\sqrt{2}}\left(|++\rangle_{t}+i|--\rangle_{t}\right),
$$

where $| \pm\rangle_{t}=\left(|0\rangle_{t}+|1\rangle_{t}\right) / \sqrt{2}$. Note that $A(\tau)=-\pi / 2$ can be achieved by choosing $v=2 \lambda_{2} \sqrt{k}$, and thus the gate time is $\tau=\sqrt{k} \pi / \lambda_{2}$. It is noted that the gate time will be increased for larger $k$, which leads to more severe decoherence effect. Therefore, $k=1$ is usually adopted. 
We next consider the influence of dissipation to the entangling gate by integrating the quantum master equation,

$$
\begin{aligned}
\dot{\rho}= & -i\left[H_{I}, \rho\right]+\kappa\left(2 a \rho a^{\dagger}-a^{\dagger} a \rho-\rho a^{\dagger} a\right) \\
& +\gamma \sum_{j=1}^{2}\left(2 \tau_{j}^{-} \rho \tau_{j}^{+}-\tau_{j}^{+} \tau_{j}^{-} \rho-\rho \tau_{j}^{+} \tau_{j}^{-}\right),
\end{aligned}
$$

where $\rho$ is the density matrix of the combined system of the topological qubit and cavity photon and $\kappa$ and $\gamma$ are the decay rate of the cavity and the lifetime of the topological qubit, respectively. We characterize the entanglement generation process by the conditional fidelity of the quantum state defined by $F=\left\langle\psi_{f}\left|\rho_{a}\right| \psi_{f}\right\rangle$, with $\rho_{a}$ being the reduced density matrix of the topological qubits. In Fig. 2, we plot the fidelity $F$ with $k=1$ as a function of dimensionless time $\lambda_{2} t / \pi$, where we have obtained high fidelity $F \simeq 95 \%$ for the generation. In the plot, we have chosen the conservative parameters of $\kappa=\gamma=1 \mathrm{MHz}$. Although the coherence time of the topological qubit may be longer, we still choose $\gamma=1 \mathrm{MHz}$ as Hamiltonian (11) is mediated by the superconducting qubit and $1 \mu \mathrm{s}$ is much shorter than its coherence time [24].

In summary, we have proposed to implement tunable interfaces between the topological qubit and the superconducting charge qubit or the microwave cavity. Combining the two interfaces, we are able to have control over and read out a topological qubit as well as implement nontrivial entangling gates between two different qubits. Therefore, the two interfaces constitute the building blocks towards universal quantum computation with topological qubits.

This work was supported by the NSFC (Grants No. 11004065, No. 11125417, No. 11105136, and No. 11104096), the NFRPC (Grants No. 2013CB921804 and No. 2011CB922104), the PCSIRT, the GRF (Grants No. HKU7058/11P and No. HKU7045/13P), the CRF (Grant No. HKU-8/11G) of the RGC of Hong Kong, and the URC fund of HKU.
[1] A. Kitaev, Ann. Phys. (NY) 303, 2 (2003).

[2] C. Nayak, S. H. Simon, A. Stern, M. Freedman, and S. Das Sarma, Rev. Mod. Phys. 80, 1083 (2008).

[3] X.-L. Qi and S.-C. Zhang, Rev. Mod. Phys. 83, 1057 (2011).

[4] M. Z. Hasan and C. L. Kane, Rev. Mod. Phys. 82, 3045 (2010).

[5] L. Fu and C. L. Kane, Phys. Rev. Lett. 100, 096407 (2008).

[6] J. D. Sau, R. M. Lutchyn, S. Tewari, and S. Das Sarma, Phys. Rev. Lett. 104, 040502 (2010).

[7] J. Alicea, Phys. Rev. B 81, 125318 (2010).

[8] S.-L. Zhu, L.-B. Shao, Z. D. Wang, and L.-M. Duan, Phys. Rev. Lett. 106, 100404 (2011).

[9] J. Liu, Q. Han, L. B. Shao, and Z. D. Wang, Phys. Rev. Lett. 107, 026405 (2011).

[10] R. M. Lutchyn, J. D. Sau, and S. Das Sarma, Phys. Rev. Lett. 105, 077001 (2010).

[11] Y. Oreg, G. Refael, and F. von Oppen, Phys. Rev. Lett. 105, 177002 (2010).

[12] J. Alicea, Y. Oreg, G. Refael, F. von Oppen, and M. P. A. Fisher, Nat. Phys. 7, 412 (2011).

[13] K. Flensberg, Phys. Rev. Lett. 106, 090503 (2011).

[14] P. Bonderson and R. M. Lutchyn, Phys. Rev. Lett. 106, 130505 (2011).

[15] M. Leijnse and K. Flensberg, Phys. Rev. Lett. 107, 210502 (2011); Phys. Rev. B 86, 104511 (2012).

[16] Z.-Y. Xue, Eur. Phys. J. D 67, 89 (2013).

[17] F. Hassler, A. R. Akhmerov, C.-Y. Hou, and C. W. J. Beenakker, New J. Phys. 12, 125002 (2010).

[18] F. Hassler, A. R. Akhmerov, and C. W. J. Beenakker, New J. Phys. 13, 095004 (2011).
[19] C.-Y. Hou, F. Hassler, A. R. Akhmerov, and J. Nilsson, Phys. Rev. B 84, 054538 (2011).

[20] Z.-Y. Xue, JETP Lett. 94, 213 (2011).

[21] L. Jiang, C. L. Kane, and J. Preskill, Phys. Rev. Lett. 106, 130504 (2011).

[22] Z.-T. Zhang and Y. Yu, Phys. Rev. A 87, 032327 (2013).

[23] Z.-Y. Xue, Z.-Q. Yin, Y. Chen, Z. D. Wang, and S.-L. Zhu, arXiv:1301.4139.

[24] D. Vion, A. Aassime, A. Cottet, P. Joyez, H. Pothier, C. Urbina, D. Esteve, and M. H. Devoret, Science 296, 886 (2002).

[25] A. Cottet, D. Vion, A. Aassime, P. Joyez, D. Esteve, and M. H. Devoret, Physica C 367, 197 (2002).

[26] J. Q. You, J. S. Tsai, and F. Nori, Phys. Rev. B 68, 024510 (2003).

[27] R. J. Schoelkopf and S. M. Girvin, Nature (London) 451, 664 (2008).

[28] L. Jiang, G. K. Brennen, A. Gorshkov, K. Hammerer, M. Hafezi, E. Demler, M. D. Lukin, and P. Zoller, Nat. Phys. 4, 482 (2008).

[29] M. Aguado, G. K. Brennen, F. Verstraete, and J. I. Cirac, Phys. Rev. Lett. 101, 260501 (2008).

[30] Z.-Y. Xue, S.-L. Zhu, J. Q. You, and Z. D. Wang, Phys. Rev. A 79, 040303 (2009).

[31] Y. Makhlin, G. Schön, and A. Shnirman, Rev. Mod. Phys. 73, 357 (2001).

[32] Z.-Y. Xue and Z. D. Wang, Phys. Rev. A 75, 064303 (2007); S.-L. Zhu, Z. D. Wang, and P. Zanardi, Phys. Rev. Lett. 94, 100502 (2005).

[33] A. Sørensen and K. Mølmer, Phys. Rev. A 62, 022311 (2000).

[34] S.-L. Zhu and Z. D. Wang, Phys. Rev. Lett. 91, 187902 (2003). 